\title{
On the effective permittivity of silver-insulator nanocomposites
}

\author{
Tom G. Mackay \\ School of Mathematics, University of Edinburgh, Edinburgh EH9 3JZ, UK \\ T.Mackay@ed.ac.uk
}

\begin{abstract}
The Bruggeman formalism provides an estimate of the effective permittivity of a composite material comprising two constituent materials, with each constituent material being composed of electrically small particles. When one of the constituent materials is silver and the other is an insulating material, the Bruggeman estimate of the effective permittivity of the composite exhibits resonances with respect to volume fraction that are not physically plausible.
\end{abstract}

Keywords: Bruggeman formalism, negative permittivity, homogenization

\section{INTRODUCTION}

The estimation of the electromagnetic properties of homogenized composite materials (HCMs) is a fundamental problem which has generated a vast literature, spanning 200 years [1]. Interest in this topic has escalated lately with the advent of metamaterials [2]; i.e., artificial composite materials which exhibit properties either not exhibited at all by their constituents or not exhibited to the same extent by their constituents [3]. In particular, metamaterials in the form of nanocomposites - which are assemblies of disparate nanoparticles - present exciting possibilities.

An important category of HCM is considered in this communication: That is, we consider HCMs which arise from the homogenization of two isotropic dielectric constituent materials, namely materials $a$ and $b$ with relative permittivities $\epsilon_{a}$ and $\epsilon_{b}$, characterized by $\delta<0$, where*

$$
\delta=\frac{\operatorname{Re}\left\{\epsilon_{a}\right\}}{\operatorname{Re}\left\{\epsilon_{b}\right\}} .
$$

A recent study demonstrated that conventional homogenization formalisms, such as the Bruggeman and Maxwell Garnett formalisms, do not necessarily provide physically plausible estimates of the HCM relative permittivity in the $\delta<0$ regime [4]. Furthermore, much-used bounds on the HCM relative permittivity, such as the Hashin-Shtrikman and Bergman-Milton bounds, can become exceedingly large when $\delta<0$ [5].

Many metal-insulator HCMs of interest belong to the $\delta<0$ category, with silver often being the constituent metal of choice. A key property of silver which is exploited by designers of HCM-metamaterials is that its relative permittivity $\epsilon_{A g}$ is such that $\operatorname{Re}\left\{\epsilon_{A g}\right\}<0$ but $\operatorname{Im}\left\{\epsilon_{A g}\right\} \ll\left|\operatorname{Re}\left\{\epsilon_{A g}\right\}\right|$ at visible and near infrared wavelengths, as illustrated in Figure 1 by the plots of measured values of $\operatorname{Re}\left\{\epsilon_{A g}\right\}$ and $\operatorname{Im}\left\{\epsilon_{A g}\right\}$ provided in [6]. Whereas the Bruggeman homogenization formalism has been widely applied to estimate the relative permittivity of silver-insulator HCMs [7-13], the inherent limitations of the Bruggeman formalism in this particular $\delta<0$ scenario are not widely appreciated. Herein we highlight the possible pitfalls of applying the Bruggeman homogenization formalism to silver-insulator HCMs.

\footnotetext{
${ }^{*}$ The operators $\operatorname{Re}\{\bullet\}$ and $\operatorname{Im}\{\bullet\}$ deliver the real and imaginary parts of their complex-valued arguments.
} 


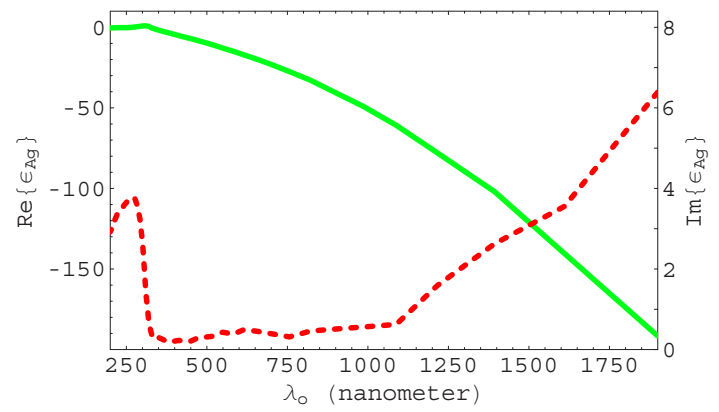

Fig. 1. Real (green, solid curve) and imaginary (red, dashed curve) parts of the relative permittivity of silver as a function of wavelength (in nanometer). From tabulated values of experimental measurements provided in [6].

\section{NUMERICAL INVESTIGATIONS}

In the Bruggeman homogenization formalism, the constituent materials are treated symmetrically. As a consequence, this approach may be applied at arbitrary volume fractions, unlike the Maxwell Garnett homogenization formalism which is restricted to dilute composites. A rigorous basis for the Bruggeman formalism is developed within the framework of the strongpermittivity-fluctuation theory (SPFT) $[14,15]$. The Bruggeman estimate of the relative permittivity of the HCM, arising from the homogenization of the two constituent materials described in $\S 1$, is provided implicitly as [16]

$$
\epsilon_{B r}=\frac{f_{a} \epsilon_{a}\left(\epsilon_{b}+2 \epsilon_{B r}\right)+f_{b} \epsilon_{b}\left(\epsilon_{a}+2 \epsilon_{B r}\right)}{f_{a}\left(\epsilon_{b}+2 \epsilon_{B r}\right)+f_{b}\left(\epsilon_{a}+2 \epsilon_{B r}\right)}
$$

wherein $f_{a}$ and $f_{b}=1-f_{a}$ are the respective volume fractions of the constituent materials $a$ and $b$. The particles of both constituent materials are assumed to be spherical and electrically small. For example, at the (optical) wavelength of 600 nanometers, constituent particles of less than 60 nanometers in radius are envisaged.

The solution

$$
\epsilon_{B r}=\frac{1}{2}\left\{-\left[\epsilon_{a}\left(f_{b}-2 f_{a}\right)+\epsilon_{b}\left(f_{a}-2 f_{b}\right)\right] \pm \sqrt{\left[\epsilon_{a}\left(f_{b}-2 f_{a}\right)+\epsilon_{b}\left(f_{a}-2 f_{b}\right)\right]^{2}+8 \epsilon_{a} \epsilon_{b}}\right\}
$$

is straightforwardly extracted from (2). The choice of sign for the square root term in (3) is dictated by the restriction $\operatorname{Im}\left\{\epsilon_{B r}\right\} \geq 0$, as per the Kramers-Kronig relations [17].

Let $\epsilon_{a}=\epsilon_{A g}$, as given by the values displayed in Figure 1. For constituent material $b$ we choose silica with $\epsilon_{b}=2.1$, in keeping with several reported Bruggeman-based studies [7, 8, 12,13]. The real and imaginary parts of the Bruggeman estimate $\epsilon_{B r}$ are plotted as functions of volume fraction $f_{a}$ in Figure 2. At the wavelengths $\lambda_{0}=397,704$ and 1393 nanometers considered in Figure 2, the corresponding values of $\epsilon_{a}$ are $-4.3+0.2 i,-23.4+0.4 i$ and $-102.0+2.6 i$, respectively [6]. The distinct resonances exhibited by $\operatorname{Im}\left\{\epsilon_{B r}\right\}$ with respect to volume fraction, and the associated abrupt changes in gradient of $\operatorname{Re}\left\{\epsilon_{B r}\right\}$, are most striking. The $\operatorname{Im}\left\{\epsilon_{B r}\right\}$ resonance range is $0 \lesssim f_{a} \lesssim 0.9$ for $\lambda_{0}=397 \mathrm{~nm}, 0.1 \lesssim f_{a} \lesssim 0.62$ for $\lambda_{0}=704 \mathrm{~nm}$ and $0.2 \lesssim f_{a} \lesssim 0.47$ for $\lambda_{0}=1393 \mathrm{~nm}$. We observe that the maximum values of $\operatorname{Im}\left\{\epsilon_{B r}\right\}$ are an order of magnitude greater than the values of $\operatorname{Im}\left\{\epsilon_{a}\right\}$ at the corresponding wavelengths.

The question arises: does the resonant behaviour illustrated in Figure 2 represent a physical process? To address this question, we repeat the calculations of Figure 2 using $\operatorname{Re}\left\{\epsilon_{a}\right\}=$ 

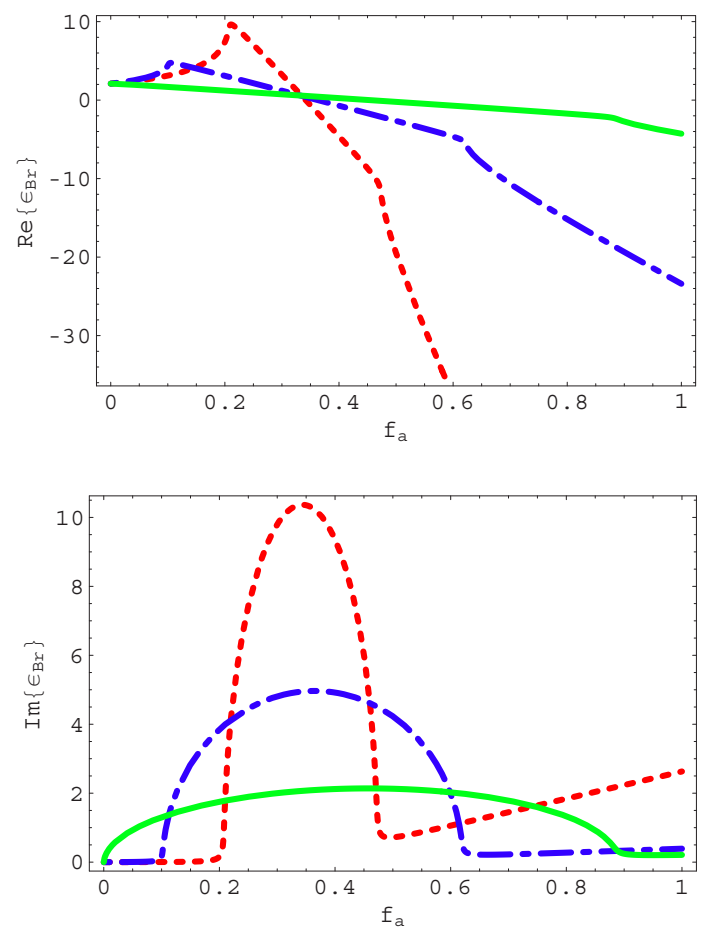

Fig. 2. The Bruggeman estimate $\epsilon_{B r}$ of the relative permittivity for the HCM arising from constituents with $\epsilon_{a}=\epsilon_{A g}$ and $\epsilon_{b}=2.1$. Real (top) and imaginary (bottom) parts are plotted against $f_{a}$ for $\lambda_{0}=1393 \mathrm{~nm}$ (red, dashed curve), $\lambda_{0}=704 \mathrm{~nm}$ (blue, broken dashed curve) and $\lambda_{0}=397 \mathrm{~nm}$ (green, solid curve).

$\operatorname{Re}\left\{\epsilon_{A g}\right\}$ but $\operatorname{Im}\left\{\epsilon_{a}\right\}=0$. The corresponding Bruggeman estimates $\operatorname{Im}\left\{\epsilon_{B r}\right\}$ are graphed against volume fraction $f_{a}$ in Figure 3 . It is clear that the resonant behaviour illustrated in Figure 2 persists in an almost identical manner even when $\operatorname{Im}\left\{\epsilon_{a}\right\}=0$. The resonances in Figure 3 are attributable to a change of sign of the radicand in (2). In the case of Figure 3 both constituent materials are nondissipative, yet the Bruggeman estimate of the HCM relative permittivity corresponds to a HCM which is strongly dissipative in the regions of resonance. However, the Bruggeman homogenization formalism has no mechanism for accommodating coherent scattering losses, unlike the SPFT which is its natural generalization [15]. Therefore, we infer that the Bruggeman estimates in the regions of resonance are not physically plausible.

\section{CONCLUDING REMARKS}

The Bruggeman estimate of the relative permittivity for a silver-insulator HCM - and, by extension, the SPFT estimate $[14,15]$ - exhibits resonances with respect to volume fraction which are not physically plausible. This point should be carefully borne in mind when considering the effective permittivity of silver-insulator nanocomposites. We note that in two recent studies wherein the Bruggeman formalism was applied to estimate the relative permittivity of silver-insulator HCMs, the resonance region was excluded from consideration $[12,13]$.

\section{Acknowledgments}

The author is supported by a Royal Society of Edinburgh/Scottish Executive Support Research Fellowship. 


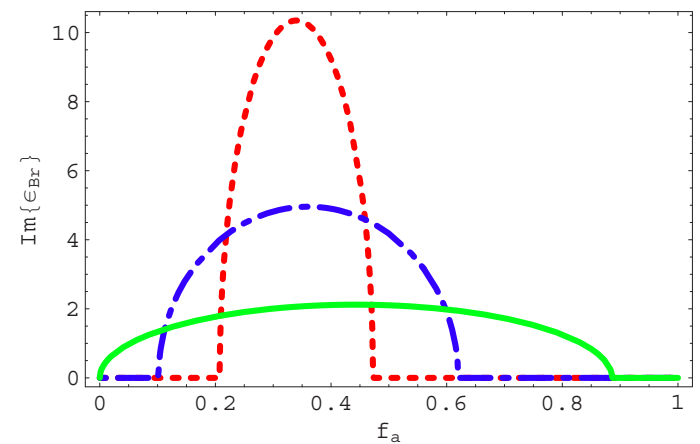

Fig. 3. The imaginary part of the Bruggeman estimate $\epsilon_{B r}$ of the relative permittivity for the HCM arising from constituent materials with $\epsilon_{a}=\operatorname{Re}\left\{\epsilon_{A g}\right\}$ and $\epsilon_{b}=2.1$, plotted against $f_{a}$ for $\lambda_{0}=1393 \mathrm{~nm}$ (red, dashed curve), $\lambda_{0}=704 \mathrm{~nm}$ (blue, broken dashed curve) and $\lambda_{0}=397$ nm (green, solid curve).

\section{References}

[1] A. Lakhtakia (Ed.), Selected Papers on Linear Optical Composite Materials, SPIE Optical Engineering Press, Bellingham, WA, USA (1996).

[2] R.M. Walser, "Metamaterials: an introduction," in Introduction to Complex Mediums for Optics and Electromagnetics, W.S. Weiglhofer and A. Lakhtakia, Eds., pp. 295-316, SPIE Press, Bellingham, WA, USA (2003).

[3] T.G. Mackay, "Linear and nonlinear homogenized composite mediums as metamaterials," Electromagnetics 25, 461-481 (2005). [doi:10.1080/02726340590957425]

[4] T.G. Mackay and A. Lakhtakia, "A limitation of the Bruggeman formalism for homogenization,” Opt. Commun. 234, 35-42 (2004). [doi:10.1016/j.optcom.2004.02.007]

[5] A.J. Duncan, T.G. Mackay, and A. Lakhtakia, "On the Bergman-Milton bounds for the homogenization of dielectric composite materials," Opt. Commun. (accepted for publication). http://arxiv.org/abs/physics/0512008.

[6] P.B. Johnson and R.W. Christy, "Optical constants of the noble metals," Phys. Rev. B 6, 4370-4379 (1972). [doi:10.1103/PhysRevB.6.4370]

[7] C.G. Granqvist and O. Hunderi, "Optical properties of $\mathrm{Ag}-\mathrm{SiO}_{2}$ Cermet films: a comparison of effective-medium theories," Phys. Rev. B 18, 2897-2902 (1978). [doi:10.1103/PhysRevB.18.2897]

[8] R.K. Roy, S.K. Mandal, D. Bhattacharyya, and A.K. Pal, "An ellipsometric investigation of $\mathrm{Ag} / \mathrm{SiO}_{2}$ nanocomposite thin films," Eur. Phys. J. B 34, 25-31 (2003). [doi:10.1140/epjb/e2003-00192-5]

[9] S.M. Aouadi, M. Debessai, and P. Filip, "Zirconium nitride silver nanocomposite structures for biomedical applications," J. Vac. Sci. Technol. B 22, 1134-1140 (2004). [doi:10.1116/1.1752905]

[10] K.P. Velikov, W.L. Vos, A. Moroz, and A. van Blaaderen, "Reflectivity of metallodielectric photonic glasses,” Phys. Rev. B 69, 075108 (2004). [doi:10.1103/PhysRevB.69.075108]

[11] J.C.G. de Sande, R. Serna, J. Gonzalo, C.N. Afonso, D.E. Hole, and A. Naudon, "Refractive index of Ag nanocrystals composite films in the neighborhood of the surface plasmon resonance," J. Appl. Phys. 91, 1536-1541 (2002). [doi:10.1063/1.1427404]

[12] W. Cai, D.A. Genov, and V.M. Shalaev, "Superlens based on metal-dielectric composites," Phys. Rev. B 72, 193101 (2005). [doi:10.1103/PhysRevB.72.193101]

[13] U.K. Chettiar, A.V. Kildishev, T.A. Klar, and V.M. Shalaev, "Negative index metamaterial combining magnetic resonators with metal films," Opt. Express 14, 7872-7877 (2006). [doi:10.1364/OE.14.007872] 
[14] L. Tsang and J.A. Kong, "Scattering of electromagnetic waves from random media with strong permittivity fluctuations," Radio Sci. 16, 303-320 (1981).

[15] T.G. Mackay, A. Lakhtakia, and W.S. Weiglhofer, "Strong-property-fluctuation theory for homogenization of bianisotropic composites: formulation," Phys. Rev. E 62, 6052-6064 (2000). [doi:10.1103/PhysRevE.62.6052] Corrections: 63, 049901 (2001). [doi:10.1103/PhysRevE.63.049901]

[16] L. Ward, The Optical Constants of Bulk Materials and Films, 2nd edition, Institute of Physics, Bristol, UK (2000).

[17] C.F. Bohren and D.R. Huffman, Absorption and Scattering of Light by Small Particles, Wiley, New York, NY, USA (1983). 